\title{
A protocol for imaging of the Drosophila visceral muscle
}

Jongkyeong Chung ( $\sim$ jkc@snu.ac.kr)

Seoul National University

\section{Soohong Min}

Seoul National University

Method Article

Keywords: Drosophila intestine, visceral muscle, imaging

Posted Date: January 25th, 2018

DOI: https://doi.org/10.1038/protex.2017.158

License: (c) (1) This work is licensed under a Creative Commons Attribution 4.0 International License. Read Full License 


\section{Abstract}

The unique properties of intestine such as intestinal stem cells, microbiome, hormonal regulations and highly specialized function for digestion and absorbing nutrients have brought much attention in this tissue for stem cell biology, endocrinology, metabolism and clinical implication for intestine-associated diseases. The Drosophila intestine preserves many aspects of the properties with simplicity, and thus this tissue has become popular among those studies. However, the muscular layers of the intestine $\backslash($ visceral muscle) have gained relatively less attention. Here we provide a protocol for preparation of the Drosophila visceral muscle for imaging analyses.

\section{Introduction}

The Drosophila visceral muscle is composed of two muscular layers comprising the very surface of the intestine: binucleated circular and multinucleated longitudinal muscles. To prepare intact structure of the visceral muscle in Drosophila, it is important to be aware of the anatomy of intestine relative to other organs prior to physical dissociation of it out of the body cavity without damaging the muscle \(Figure 1).

\section{Reagents}

Flies bearing Mef2-GAL4 and UAS-mCD8GFP \(Mef2>mCD8GFP), phosphate-buffered saline \(PBS), Hoechst $33342 \backslash$ (Thermo Fisher Scientific, H1399), 4\% paraformaldehyde \(PFA), TRITC/FITC-conjugated phalloidin \(Thermo Fisher Scientific, F432 and R415), anti-mouse myosin heavy chain antibody $\backslash$ (DSHB, ALD-58), anti-mouse tubulin antibody \(Abcam, ab6046). SlowFade mounting solution \(Invitrogen, S36936). DNase solution \(Thermo Fisher Scientific, AM2238).

\section{Equipment}

A common light microscope with $\sim 20 \mathrm{X}$ resolution, Sylgard dissecting plate, confocal microscope $\backslash$ (LSM710, Carl Zeiss). nutator, $4^{\circ} \mathrm{C}$ freezer, $37^{\circ} \mathrm{C}$ incubator, a pair of sharp forceps and microscissors $\backslash$ (Fine Science Tools).

\section{Procedure}

- Preparation of the Drosophila intestine for immunostaining on the visceral muscle 1. Decapitate wild type flies $\backslash$ (Use FITC/TRITC-conjugated phalloidin antibodies for staining visceral muscle fibers) or Mef2>mCD8GFP flies $\backslash$ (which label visceral muscle fibers) in PBS using microscissors. 2. Expose the forepart of the intestine by selective removal of legs, thoracic muscles and the thoracic junction cuticle to the abdomen using a pair of forceps. 3. Make an incision from the beginning of the abdomen to the anus without damaging the intestine. 4. Grab the anus and pull it carefully to separate the whole intestine from the abdominal cuticle. 5. Remove the reproductive organ from the dissected intestine. 6 . Incubate the intestine in 4\% PFA for 20 minutes. Nutation is optional for subsequent procedures except for the 
antibody incubation. Thorough nutation likely causes some damages of the intestine. 7. Wash the tissue by 2 times in $0.1 \%$ PBST for 5 minutes. 8 . Permeabilize the intestine by incubation in $0.5 \%$ PBST for 10 minutes. 9. Wash the tissue 3 times in $0.1 \%$ PBST for 5 minutes. If secondary antibody treatment is not required, simply proceed to adding Hoechst $\backslash(1: 400)$ and FITC/TRITC-conjugated phalloidin $\backslash(1: 200)$ in the tissue-containing solution and incubate for $\sim 2$ hours at room temperature $\backslash(R T)$ on a nutator. Next, proceed to step 13. 10. For secondary antibody treatment, incubate the tissue in $3 \%$ bovine serum albumin in $0.1 \%$ PBST for 30 minutes at RT for blocking. 11. Add primary antibodies including antimouse tubulin antibody $\backslash($ Abcam, ab6046, 1:200) and anti-mouse myosin heavy chain antibody $\backslash($ DSHB, ALD-58, 1:100), and incubate overnight at $4^{\circ} \mathrm{C}$. 12 . On the next day, wash the tissue by nutating in $0.1 \%$ PBST 3 times for 5 minutes, and the following antibodies were treated for 2 hours at RT in dark: antimouse dye-conjugated antibodies $\backslash(1: 200)$, FITC/TRITC-conjugated phalloidin antibodies $\backslash(1: 200)$ and Hoechst $\backslash(1: 400)$. 13. Wash the tissue by nutating in $0.1 \%$ PBST 3 times for 5 minutes and in PBS 1 time for 5 minutes. 14. Place the whole tissue on a slide glass and mount it in SlowFade solution $\backslash$ (Invitrogen, CA, ID: S36936) with a cover glass. - Preparation of the Drosophila intestine for imaging of apoptotic responses on the visceral muscle 1. Perform the same steps 1-6 described in the above tissue preparation section. 2. Wash the tissue 2 times in $0.5 \%$ PBST for 10 minutes and discard the washing solution. 3 . Add $0.1 \mathrm{M}$ sodium citrate in $0.1 \% \mathrm{PBST}$ and incubate the tissue at $65^{\circ} \mathrm{C}$ for 30 minutes. 4 . Wash the tissue with PBS 2 times for 10 minutes. 5. As a control group, add DNase solution $\backslash(1.5 \mu l$ of DNase, $3 \mu l$ of 10 $\mathrm{mg} / \mathrm{ml} \mathrm{BSA}, 1.5 \mu \mathrm{l}$ of $1 \mathrm{M}$ Tris buffer with $\mathrm{pH} 7.5$, and $24 \mu \mathrm{l}$ of $\mathrm{H} 2 \mathrm{O})$. 6 . For TUNEL reaction, incubate the experimental samples in $2 \mu \mathrm{l}$ of TUNEL enzyme and $18 \mu \mathrm{l}$ of buffer at $37^{\circ} \mathrm{C}$ for 2 hours. 7 . Wash the tissue in $0.1 \%$ PBST for 10 minutes and incubate with phalloidin-TRITC/FITC and Hoechst for 1 hour at RT. 8. Wash the samples 3 times in $0.1 \%$ PBST for 10 minutes. 9. Mount the sample for confocal imaging as described in the previous immunostaining section. - Confocal imaging of the visceral muscle and measuring the diameter of the intestine 1. Obtain Z-stack images of the intestine using a confocal microscope \(LSM710, Carl Zeiss). When taking the Z-stack images, pay attention to the visceral muscle layers which could be skipped easily for its shallow structure on the surface of the intestine. The characteristic multinuclei of the visceral muscle can be a hallmark to distinguish the muscle from the intestinal cells which also appear after the visceral muscle in the Z-stack images. 2. Make a projection image using the Z-stack images and measure width of a few regions of the intestine as a representative diameter of the given intestine.

\section{Anticipated Results}

- The structures of the visceral muscle can be well visualized by immunostaining using antibodies against phalloidin $\backslash$ (F-actin), tubulin and myosin $\backslash$ (Figure 2$)$. - The apoptosis in the visceral muscle can be imaged by TUNEL labeling of the muscle $\backslash($ Figure 3). - The diameter of the intestine can be represented by averaging the width of a few regions of the Z-stacked intestine images $\backslash$ (Figure 4).

\section{Acknowledgements}


This research was supported by a grant $\backslash(\mathrm{HI} 17 \mathrm{C} 0328)$ of the Korea Health Technology R\&D project through the Korea Health Industry Development Institute $\backslash(\mathrm{KHIDI})$, funded by the Ministry of Health \& Welfare.

\section{Figures}

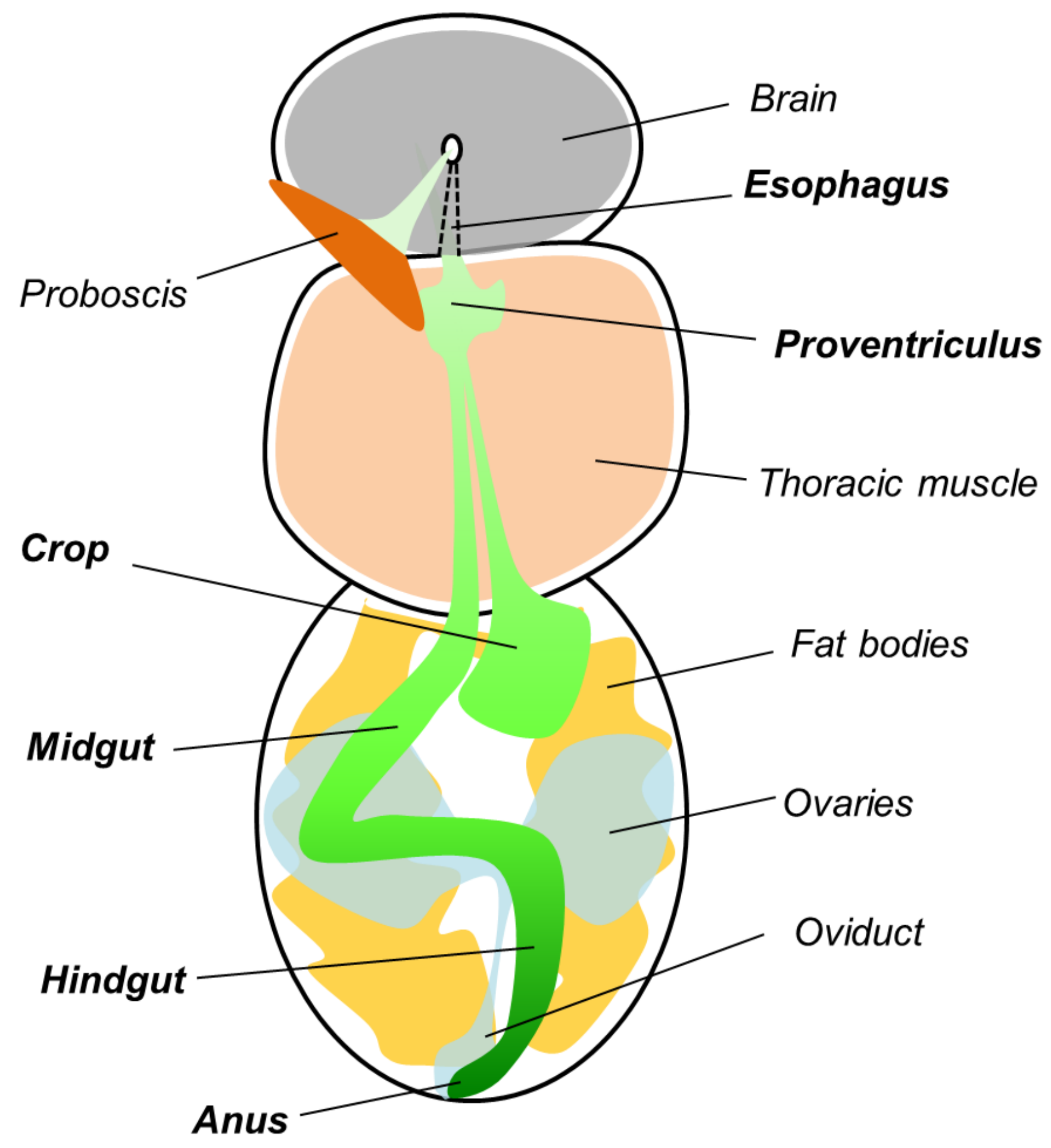

Figure 1 
Anatomy of the Drosophila organs Bold italic words indicate the intestinal parts. Note the relative location of the parts in comparison to other organs.
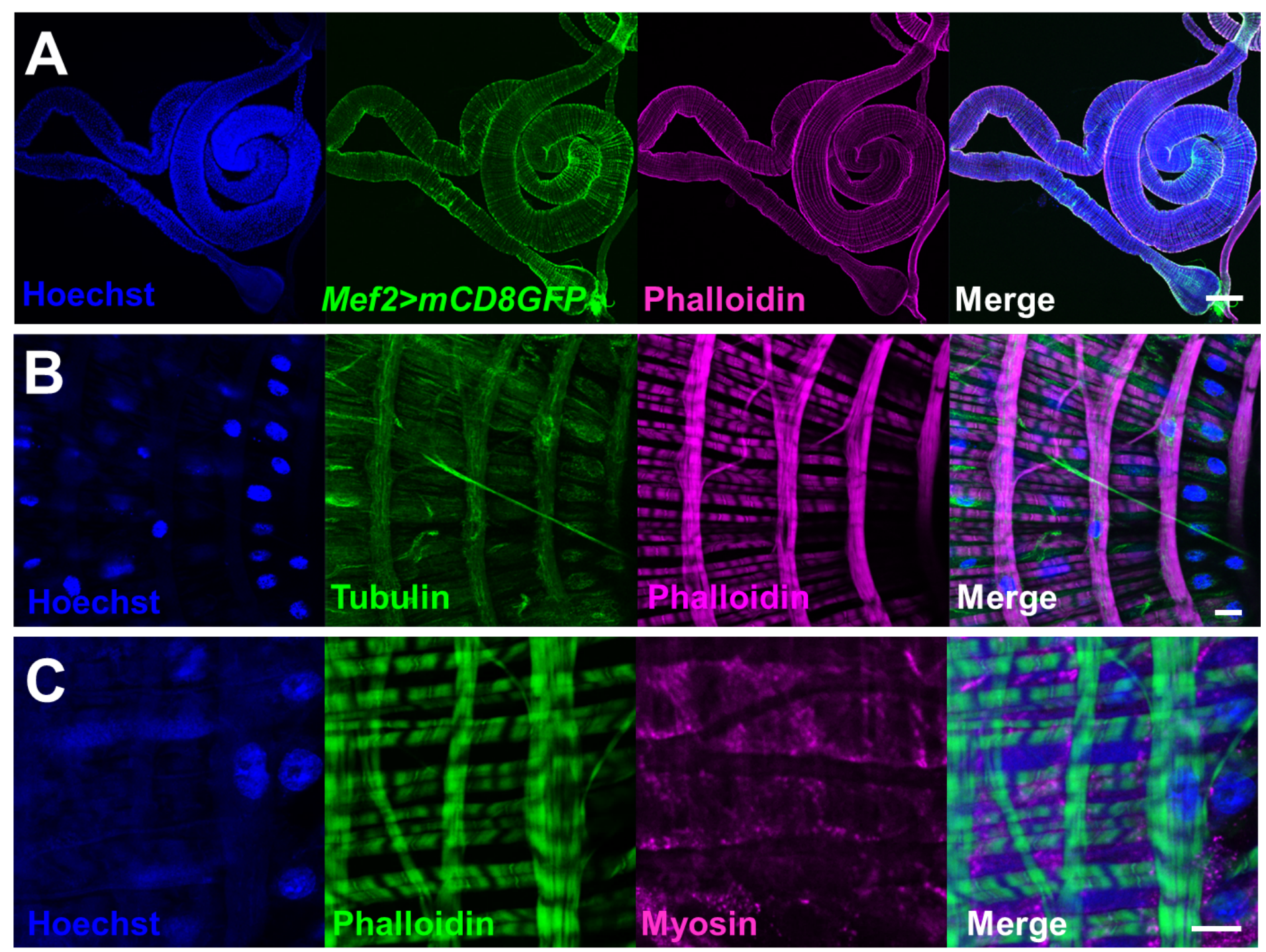

\section{Figure 2}

Confocal imaging of the stained visceral muscle The structures of the visceral muscle were visualized using antibodies against phalloidin (A), tubulin (B) and myosin (C) or a genetic labeling of the visceral muscle by Mef2-GAL4-driven mCD8GFP expression. Panels $B$ and $C$ are the images shown in the associated paper with some modifications. Scale bars for A: $200 \mu \mathrm{m}$; for B and C: $10 \mu \mathrm{m}$. 


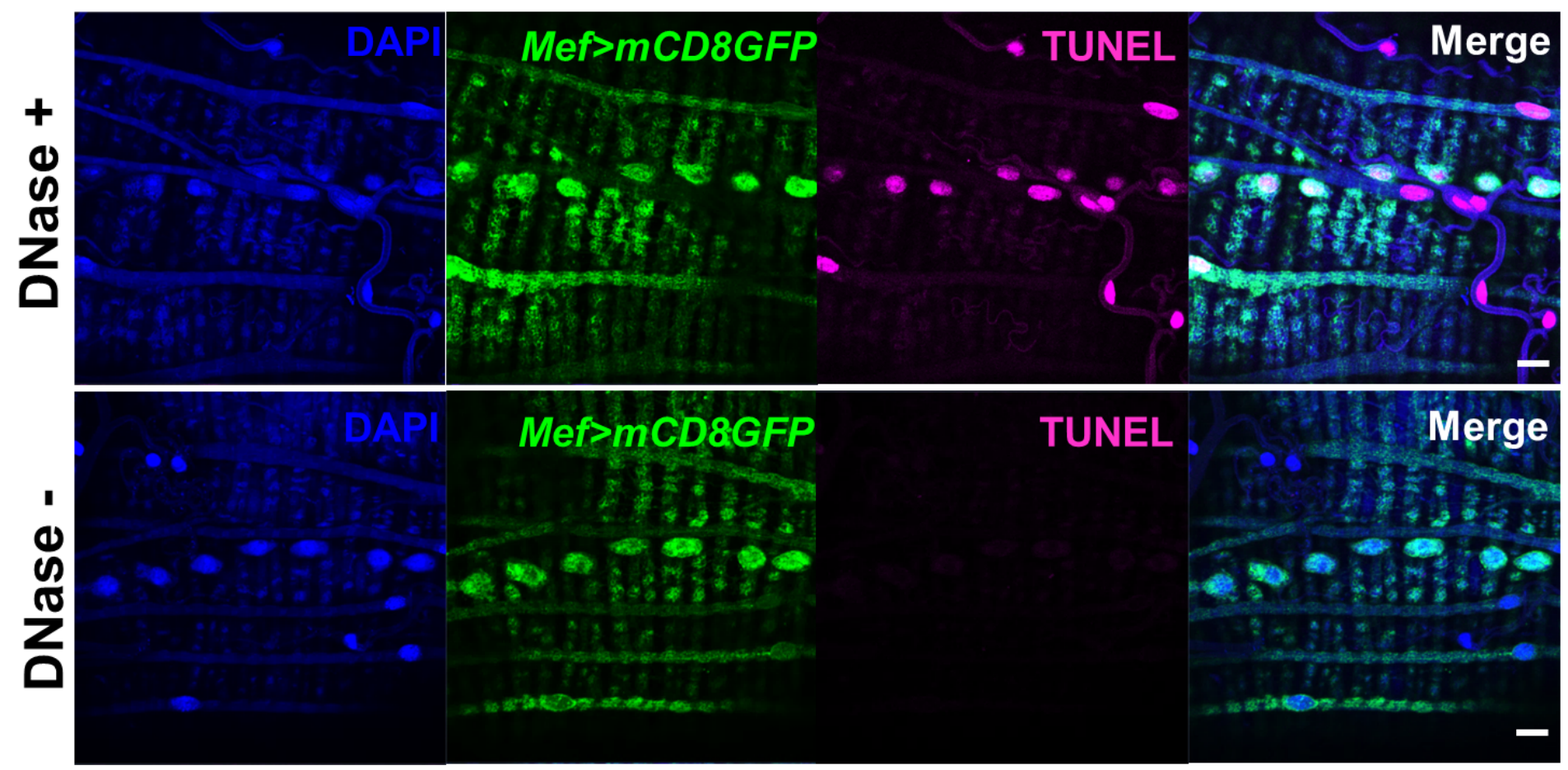

Figure 3

Imaging of apoptotic responses on the visceral muscle The DNase-treated (DNase + ) visceral muscle showed marked apoptotic responses as opposed to non-treated (DNase -) muscle. These are the images shown in the associated paper with some modifications. Scale bars: $10 \mu \mathrm{m}$. 


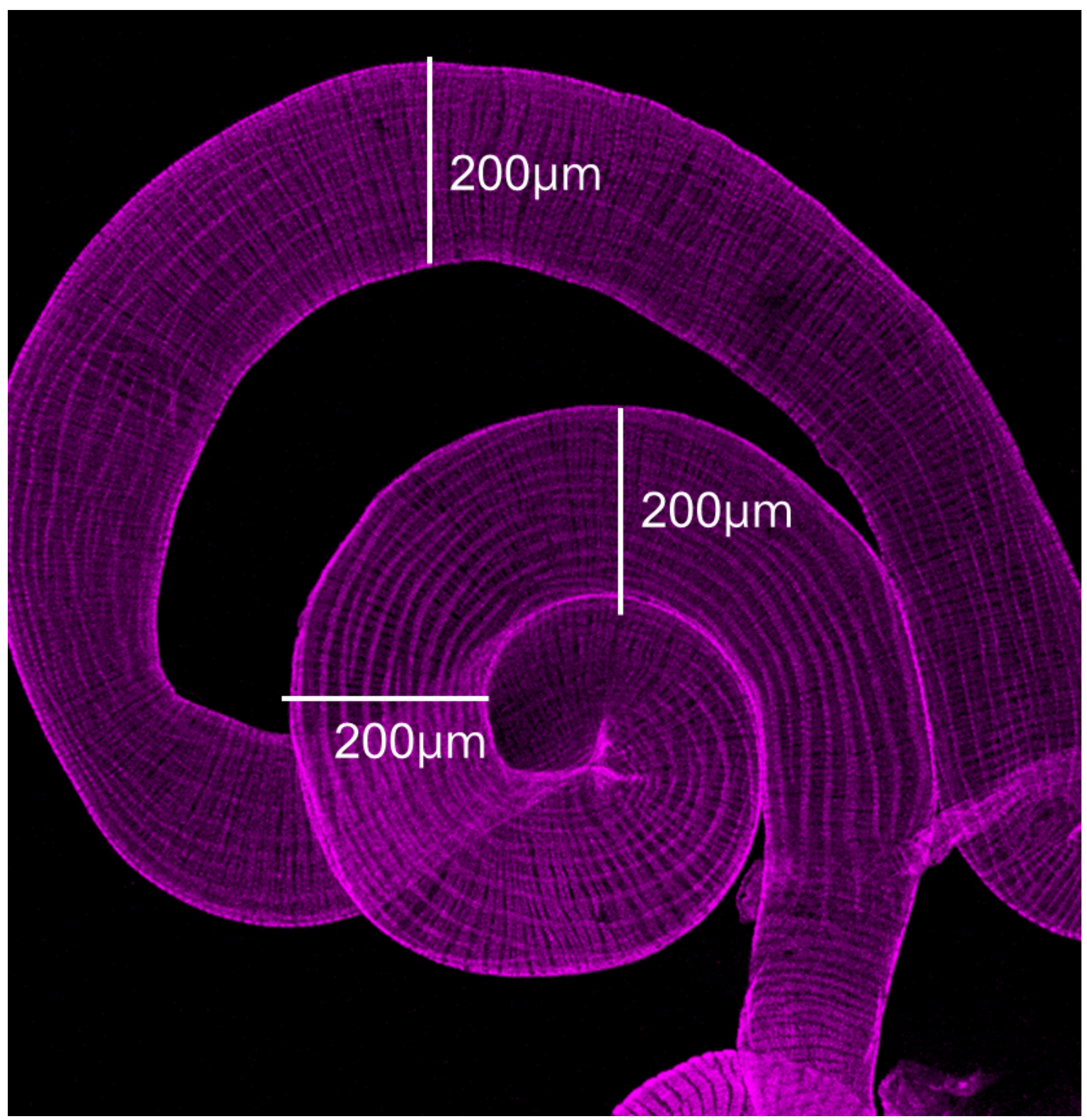

Figure 4

Measurement of the diameter for the intestine The image of the Drosophila intestine is a projection of the Z-stack images of the intestine stained with anti-phalloidin antibody. 